\title{
RINGS: \\ A Technique for Visualizing Large Hierarchies
}

\author{
Soon Tee Teoh and Kwan-Liu Ma \\ Computer Science Department, \\ University of California, Davis \\ \{teoh, ma\}@cs.ucdavis.edu
}

\begin{abstract}
We present RINGS, a technique for visualizing large trees. We introduce a new ringed circular layout of nodes to make more efficient use of limited display space. RINGS provides the user with the means to specify areas of primary and secondary focus, and is able to show multiple foci without compromising understanding of the graph. The strength of RINGS is its ability to show more area in focus and more contextual information than existing techniques. We demonstrate the effectiveness of RINGS by applying it to the visualization of a Unix file directory.
\end{abstract}

\section{Introduction}

Ringed Interactive-Navigation Graph System (RINGS) is a technique for visualizing large hierarchical data, as well as large graphs if a spanning tree of the graph is provided as input. RINGS is based on a new ringed circular tree layout algorithm described in more detail in Section 2. Existing methods such as [3 5/7] tend to leave large regions of space empty. Shneiderman's Tree-maps [8] method makes efficient use of screen space to show a large number of nodes, but in the Tree-map layout, the structural information of the trees is unclear. Three-dimensional visualization methods such as Munzner's hyperbolic tree [5] and Kleiberg's botanical tree 2] can be used to navigate through very large graphs and trees, respectively. However, overlapping of edges due to projecting a three-dimensional graph onto a two-dimensional space can lead to occlusion and ambiguity, limiting their effectiveness.

The main advantage of the ringed circular method is its efficient use of limited display size by showing more distinguishable nodes at one time, without any of the other methods' shortcomings listed above. Another important feature of RINGS is its capability to show more contextual information without compromising the clarity of the area in focus. Besides providing interactive navigation and focus+context solutions common in many existing systems, RINGS can also show areas in secondary focus effectively. We also describe how RINGS handles the problem of edge crossing and occlusion.

Graphs are often used to display embedded information. RINGS is conducive for this purpose. In Section 5, we demonstrate how RINGS can be used to visualize file sizes, locate specific files and types of files in a directory, and reveal other aspects of the file directory structure. 


\section{Layout Algorithm}

Recent graph-drawing algorithms such as Kreuseler et al.'s tree on a hemisphere [3] and Munzner's hyperbolic tree [5] provide good navigation and focus+context solutions, but they are often less effective in using the available screen space. With a $512 \times 512$ display, typically less than 100 nodes and edges are distinguisable at one time while the rest of the graph appears clumped together. In RINGS, we make better use of the available screen space, showing more distinguishable edges and also more structural information in context.

In RINGS, a node and all its children are placed in a circle. In this way, the branch any node belongs to is easily identified. Representing each node by a circle has been described in 1146]; however, they place children only on the circumference of the parent circle, wasting empty space in the interior. In the new ringed layout used by RINGS, equal-sized circles corresponding to children are placed in concentric rings around the center of the parent circle (see Figure 1).
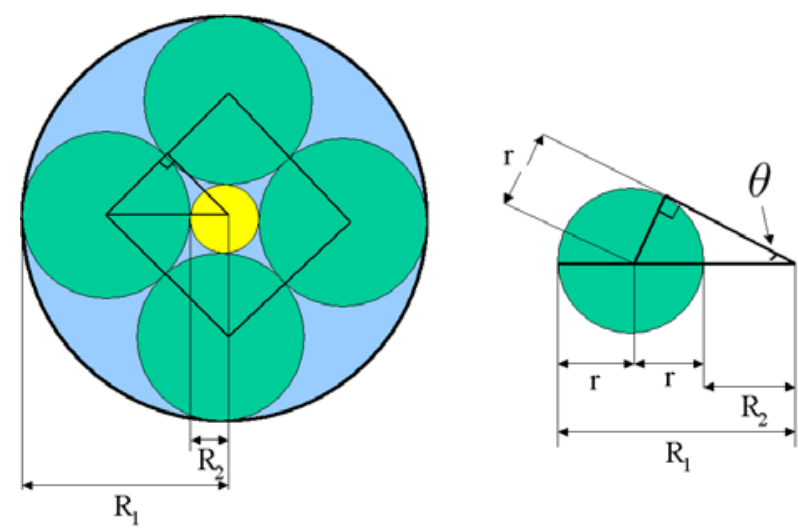

Fig. 1. Ringed circular layout of nodes: The four green circles represent the four largest children of the parent node, represented by the large blue circle. The small yellow inner circle is the area left for the remaining children.

Connecting the centers of $n$ equal circles placed in a ring makes an $n$-sided regular polygon. In Figure 1, $\theta=\pi / n$, where $n$ is the number of circles in a ring, and $\theta$ is in radians. A simple relationship can be derived between the number of children circles in the outermost ring and the percentage of area taken up by the ring. $f(n)$, the fraction of the area left after $n$ circles have been placed in the ring is given by:

$$
f(n)=\frac{\left(R_{2}\right)^{2}}{\left(R_{1}\right)^{2}}=\frac{(1-\sin (\theta))^{2}}{(1+\sin (\theta))^{2}}=\frac{\left(1-\sin \left(\frac{\pi}{n}\right)\right)^{2}}{\left(1+\sin \left(\frac{\pi}{n}\right)\right)^{2}} .
$$

This relationship is used to determine the number of children to be placed in each concentric ring. The algorithm first sorts the children by their number of 


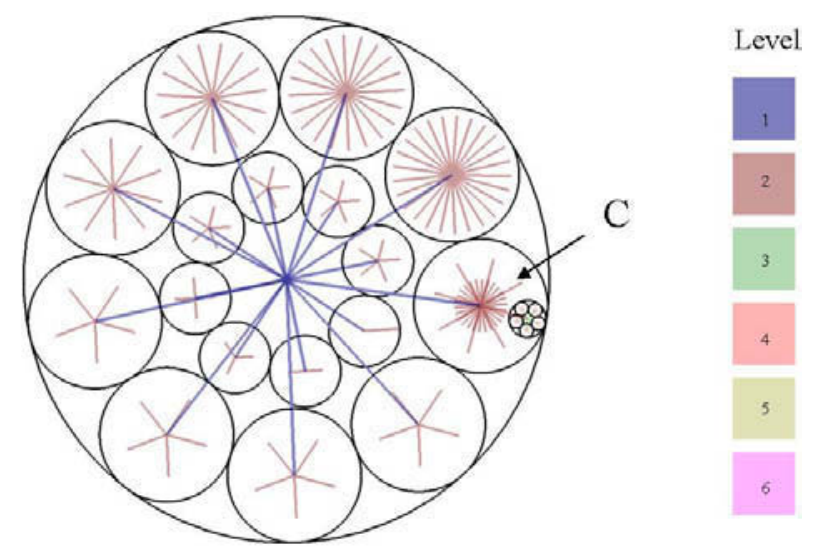

Fig. 2. Each node is placed at the center of its circle, and edges are drawn between each pair of connected nodes. Edges are colored according to their distance from the root. Level 1 edges are those directly connected to the root.

children. Next, the algorithm finds the smallest $k$ for which the sum of the number of children of the first $k$ children expressed as a fraction of the total number of grandchildren is greater or equal to $f(k)$. The first $k$ children are placed in the outermost ring. The rest of the children are placed in the same way in the inner rings. A node is positioned in the center of its circle. Edges are drawn as straight lines connecting nodes, as shown in Figure 2. The child labelled C itself has 35 children, and one of those children has descendents, whose circles are outlined in the figure. By placing the largest children in the outermost ring, the number of children in the outer rings is minimized, thereby minimizing the amount of occlusion due to edges drawn from the parent to the children.

\section{Interactive Navigation}

Like other graph visualization systems, RINGS allows the user to interactively explore the large data. The primary focus is changed by clicking on the child to focus on. The child will be moved to the center and the parent moved to the side as a smooth animation. Figure 3 demonstrates change of focus performed on a 20,000-node Unix file directory. The child in focus becomes the pictorial root because of its central position, and its true parent becomes a pictorial child, since it is placed in a circle on a ring just as any child is.

Secondary foci is set by clicking on a child to focus on and dragging it to another child currently occupying a larger area. The positions of the two children are swapped, again with smooth animation (see Figure 4 ). In addition, the user can reduce the number of children in the outermost ring so that the secondary focus can be enhanced, also shown in Figure 4 The ability to effectively show secondary foci is generally not found in existing methods. 


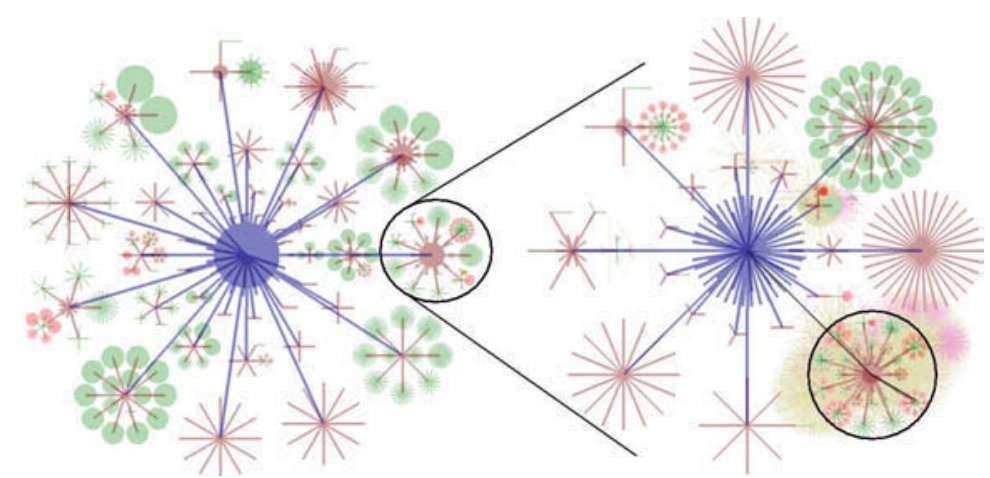

Fig. 3. Primary focus is changed by moving a child to the center. The circled child in the left drawing is expanded to the center in the right drawing, while the rest of the graph is moved to the circle in the right drawing. The edge from the focus to its true parent is colored black to distinguish it from the other edges. RINGS smoothly animates these changes.

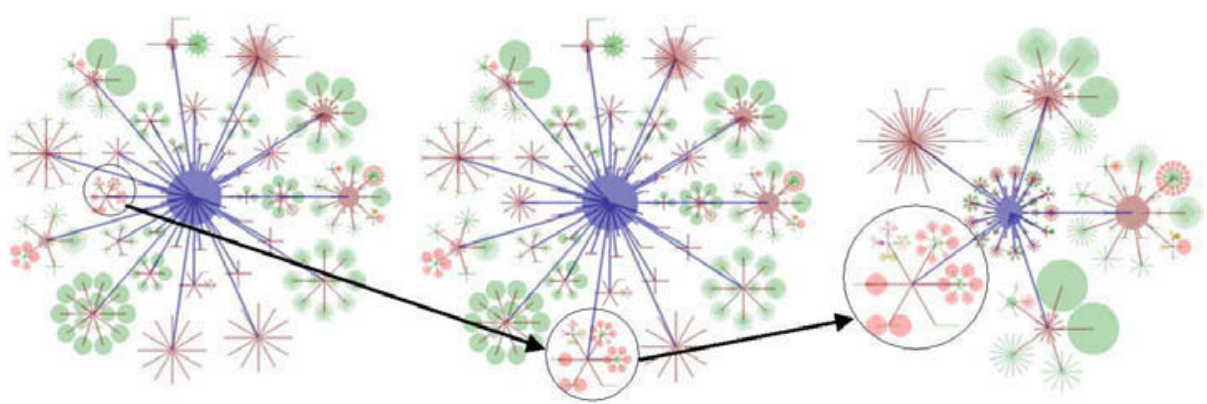

Fig. 4. Secondary focus is changed by swapping two children. In this example, the user wants to increase the focus on the circled child in the left picture. It is swapped with the selected child at the bottom, resulting in the center picture. To further increase the secondary focus, the user can reduce the number of children in the outermost ring, giving each child more screen space. The number of children is reduced from eleven in the center to five in the right picture.

\section{Other Features}

Nodes at larger topological distances from the pictorial root occupy very little screen area, so they may become indistiguisable from neighboring nodes. Many edges far away from the foci map to the same pixels on the screen. Figure 5 shows how RINGS provides the option of expanding those nodes out of the confines of their circle. This additional contextual information gives the user a sense of the depth and topology of particular branches without compromising the clarity of the regions in focus. 

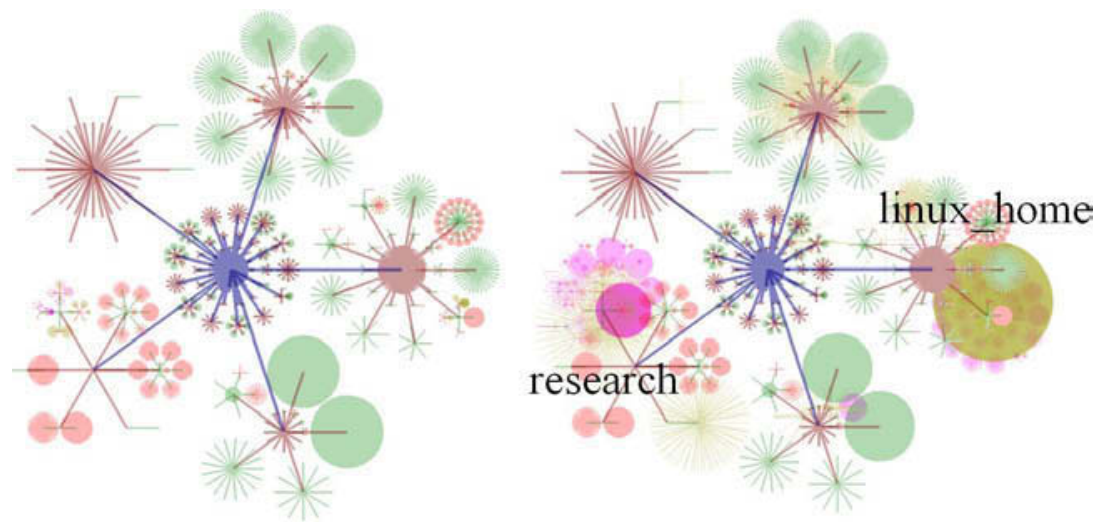

Fig. 5. The drawing on the right shows circles at topological distance 4 from the root expanded 200 times. A better sense of topology is gained without losing focus. The depth of certain sub-directories under "research" and "linux_home" is revealed.
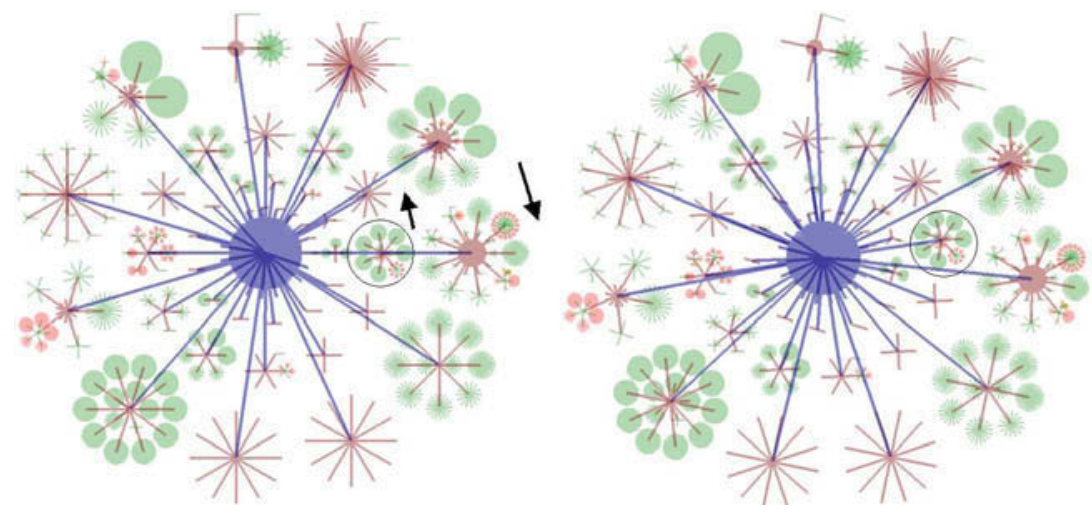

Fig. 6. Rotation: Occlusion of particular nodes can be eliminated by rotating adjacent rings in opposite directions.

A simple way to improve topology perception is to use different colors for edges with different topological distances from the root. In the color map (shown in Figure 21) used in our examples, darker, more saturated colors are used for edges closer to the root to highlight them. With the RINGS layout, occluded edges are topologically further away from the focus, so they are relatively unimportant. Nevertheless, to overcome the inevitable occlusion, edges are drawn as semi-transparent lines. In addition, RINGS allows the user to rotate adjacent concentric rings in opposite directions (see figure 6) to reveal a particular occluded branch.

When visualizing very large graphs, it becomes computationally very expensive to traverse every node in the graph and render individual edges. To maintain 
navigation at interactive rate, RINGS first creates texture maps of each branch. During interactive navigation of the graph, branches are rendered as texturemapped polygons whereever possible. In this way, even very large trees can be rendered at interactive rates.

\section{$5 \quad$ Visualizing Information Beyond Topology}

Figure 7 shows the application of RINGS to the visualization of a Unix file directory. Edges in the graph are colored according to the size of the file or directory. The two pictures show how varying the color map can be used to explore the data.

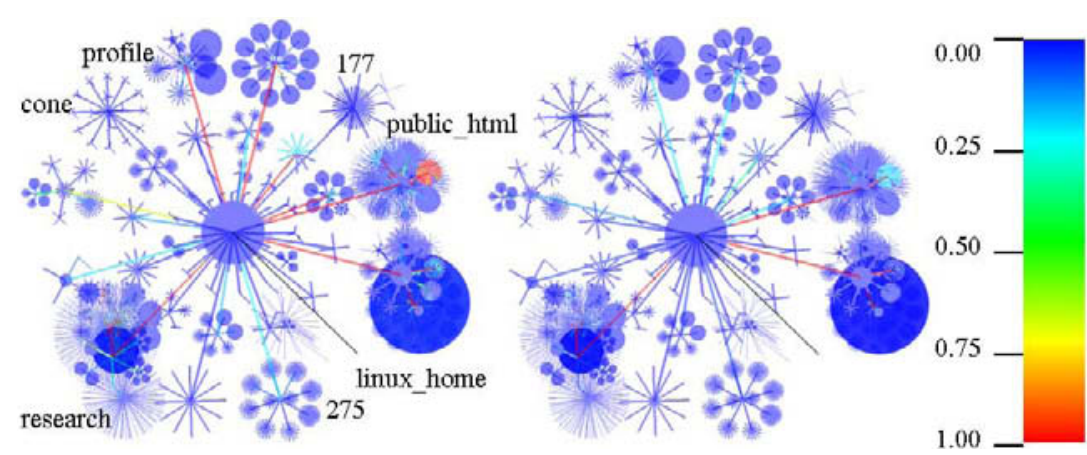

Fig. 7. Visualizing a Unix directory: In the left picture, the colormap value 1.0 is clipped at 0.1 of the total size of all files in the directory. In the right picture, it is clipped at 0.01 .

Other information in the data can also be visualized. Figure 8 shows the result of searching for a file "texture.tga". The entire path to the file is highlighted in red. In this picture, the user can see the location of the file in relation to the whole directory. The branch and sub-branches the file belongs to are also revealed. Figure 8 highlights all the files in the directory tree with ".cpp", ".cc", ".c" or ".h" extensions. This identifies all the sub-directories containing those $\mathrm{C}++$ source files. Examining individual sub-directories of Figure 8 gives insight into their internal structure. Examples of interpreting the visualization are explained in Figure 9

By visualizing typical ringed circular graphs, we can classify the patterns observed (see Figure 10). Understanding this classification aids the gaining of insights into the visualization.

\section{Conclusion}

We have presented a new ringed circular layout algorithm to visualize large graphs. The main contribution of RINGS is its efficient use of limited display 

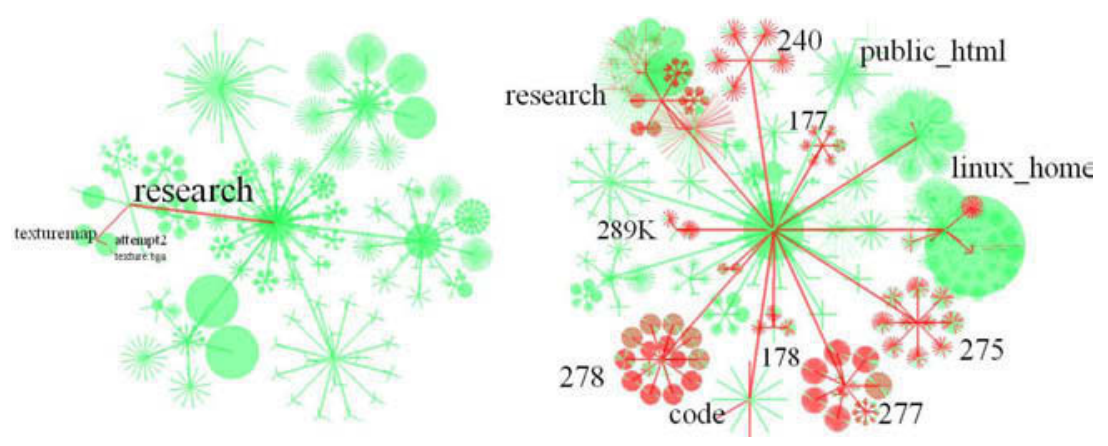

Fig. 8. Left: Visualizing the location of a particular file with respect to its neighbors, the branch it belongs to, and the entire directory tree. Right: Highlighting all $\mathrm{C}++$ source files and the directories containing them.
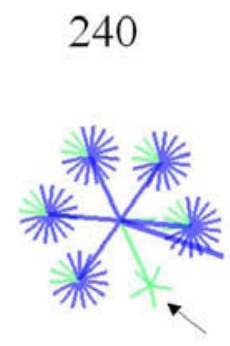

278

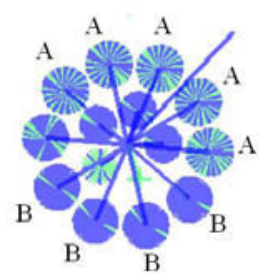

code

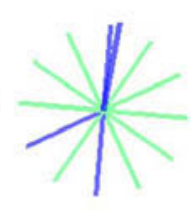

277

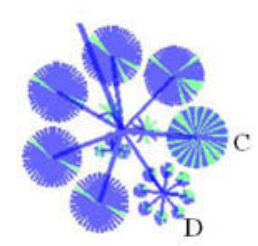

Fig. 9. Interpretation of sub-directories from Figure 8 : Sub-directory "240" has 6 subdirectories, 5 of which have a very similar structure. The arrowed one is the exception. " 278 " has many more sub-directories than " 240 " and some of them are similar, for example those labelled "A" and "B". "code" has children which are leaves (files). " 277 " has children of different sizes. 5 of the largest children are similar, but the child labelled "C" is slightly different in the composition of $\mathrm{C}++$ source files and the child labelled "D" is topologically different from the rest.

space by showing more distinguishable nodes at one time. Other important features of RINGS include showing more contextual information without compromising the clarity of the area in focus, and showing areas in secondary focus.

We have demonstrated the effectiveness of RINGS in visualizing specific information embedded in a Unix file directory. RINGS is able to highlight subdirectories with large files and/or large number of files. RINGS is also able to locate specific files and show where they are in relation to the rest of the directory.

RINGS is a general graph visualization technique, not limited to the visualization of directory trees. In the future, we plan to apply the technique to the visualization of a variety of hierarchical data, as well as general graphs with spanning trees. 
A

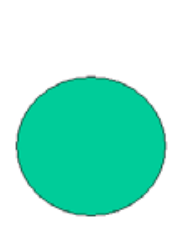

B

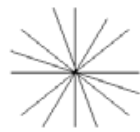

$\mathrm{C}$

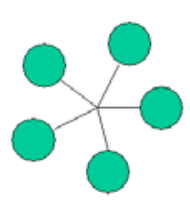

$\mathrm{D}$

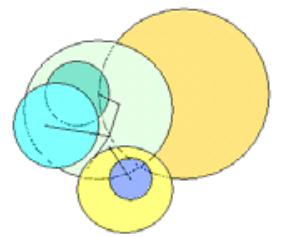

Fig. 10. Classification of basic patterns: A) A Solid circle indicates that this node has numerous children of similar size. B) A pattern of radiating lines indicates that the children are leaves. C) Radial lines with large end-points indicate that children are not leaves. D) Many overlapping circles and lines in the expanded view indicate that this node has many very deep descendents. In practice, most nodes in a graph exhibit mixed patterns. However, this simple classification allows intuitive interpretation.

\section{References}

1. E.H. Chi and S.K. Card. Sensemaking of Evolving Web Sites Using Visualization Spreadsheets. Proceedings of the Symposium on Information Visualization (Info Viz'99), pp. 18-25, 142. IEEE Press, 1999.

2. E. Kleiberg, H. van de Wetering and J.J. van Wijk. Botanical Visualization of Huge Hierarchies. Proceedings of the Symposium on Information Visualization (InfoViz'01), pp. 87-94. IEEE Press, 2001.

3. M. Kreuseler, N. Lopez and H. Schumann. A Scalable Framework for Information Visualization. Proceedings of the Symposium on Information Visualization (InfoViz'00), pp. 27-36. IEEE Press, 2000.

4. G. Melancon and I. Herman. Circular Drawing of Rooted Trees. Reports of the Center for Mathematics and Computer Sciences, Report number INS-9817, available at: http://www.cwi.nl/InfoVisu/papers/circular.pdf, 1998.

5. T. Munzner. H3: Laying out Large Directed Graphs in 3D Hyperbolic Space. Proceedings of the IEEE Symposium on Information Visualization (Info Viz'97), IEEE CS Press, pp. 2-10, 1997.

6. G.G. Robertson, J.D. Mackinlay and S.K. Card. Cone Trees: Animated 3D Visualization of Hierarchical Information. Human Factors in Computing Systems, CHI'91 Conference Proceedings, ACM Press, pp. 189-194, 1991.

7. M. Sarkar and M.H. Brown. Graphical Fisheye View of Graphs. Human Factors in Computing Systems (CHI'92) Conference Proceedings, ACM Press, pp. 83-91, 1992.

8. B. Shneiderman. Tree Visualization with Tree-maps: A 2D space-filling approach. ACM Transactions on Graphics, 11(1):92-99, September 1990. 